\title{
Resonant Raman scattering in mercurate single crystals
}

\author{
Y. Gallais ${ }^{1}$, A. Sacuto ${ }^{1,2}$ and D. Colson ${ }^{3}$ \\ 1 Laboratoire de Physique du Solide (UPR 5 CNRS) ESPCI, \\ 10 rue Vauquelin 75231 Paris. \\ 2 Matériaux et Phénomènes Quantiques (FER 2437 CNRS), Université Paris 7 \\ 2 place Jussieu 75251 Paris. \\ 3 Service de Physique de l'Etat Condensée, CEA-Saclay,91191 \\ Gif-sur-Yvette, France
}

\begin{abstract}
We report resonant electronic Raman scattering in optimally doped single layer $\mathrm{HgBa}_{2} \mathrm{CuO}_{4+\delta}(\mathrm{Hg}-1201)$ and trilayer $\mathrm{HgBa}_{2} \mathrm{Ca}_{2} \mathrm{Cu}_{3} \mathrm{O}_{8+\delta}(\mathrm{Hg}-1223)$ single crystals. Analysis of the $B_{1 g}$ and $\mathrm{B}_{2 g}$ channels in the superconducting state of $\mathrm{Hg}$-1201 advocates for a gap having d-wave symmetry. In addition a resonant study $B_{1 g}$ pair-breaking peak and the $A_{1 g}$ peak suggests that the $A_{1 g}$ peak is not directly related to the d-wave superconducting gap amplitude. Comparison with trilayer $\mathrm{Hg}-1223$ demonstrates the universal behavior of this two energy scales in optimally doped cuprates.
\end{abstract}

\section{Introduction}

In recent years electronic Raman scattering (ERS) has been used extensively to probe both the normal and the superconducting states of the cuprates. While the broad Raman continuum in the normal state is only poorly understood at present, Raman spectra in the superconducting state have been described quite successfully in terms of BCS theory with a d-wave gap in optimally doped cuprates $[1,2]$. By using different incident and scattered light polarisation ERS probes different directions in reciprocal space allowing an analysis of the gap symmetry. The $B_{1 g}$ symmetry is sensitive to the $k_{x}=0$ and $k_{y}=0$ directions and thus gives a pair-breaking peak at $2 \Delta_{0}$ while the $B_{2 g}$ symmetry probes the $k_{x}=k_{y}$ directions and is sensitive to the nodes of the d-wave gap. Although spectra taken in these two symmetries cuprates are consistent with a d-wave gap in most optimally doped cuprates, spectra taken in the fully symmetrical $\mathrm{A}_{1 g}$ channel reveal a peak below $2 \Delta_{0}$ whose intensity and position are difficult to reconcile with a simple d-wave gap picture [3]. In this symmetry, vertex corrections due to Coulomb interaction and charge conservation (or backflow) are expected to strongly suppress the scattering intensity in the superconducting state [4]. Therefore the intensity in the $A_{1 g}$ channel is expected to be much smaller than in the $B_{1 g}$ symmetry. This contradicts experimental results in most cuprates which show an $A_{1 g}$ peak intensity stronger than the $B_{1 g}$ one in most cases $[1,2,5]$. Many scenarios have been proposed to explain this discrepancy: coupling between adjacent $\mathrm{CuO}_{2}$ planes [3], resonant effect of the Raman vertex [6] and, for three and four layer cuprates, contribution from the caxis bilayer plasmon observed in the far-infrared spectra of the c-axis conductivity [7]. Recently we have shown using Ni substitution in optimally doped Y-123 that the energy of the $A_{1 g}$ peak follows intriguingly the energy of the neutron resonance detected in the spin excitation spectrum by Inelastic Neutron Scattering (INS) [5]. However the exact microscopic mechanism of the $A_{1 g}$ peak remains unclear and more experimental work is clearly needed. In this paper we report Raman scattering in $\mathrm{HgBa}_{2} \mathrm{CuO}_{4+\delta}(\mathrm{Hg}-1201)$ and $\mathrm{HgBa}_{2} \mathrm{Ca}_{2} \mathrm{Cu}_{3} \mathrm{O}_{8+\delta}(\mathrm{Hg}-1223)$ where the $2 \Delta_{0}$ pair breaking peak and the $\mathrm{A}_{1 g}$ peak have been studied as a function of the excitation energy. Both energy scales show very different resonant behaviors in Hg-1201, suggesting different underlying microscopic mechanisms. Comparison with the trilayer $\mathrm{Hg}-1223$ demonstrates 
that the nature of the two energy scales is independant of the number of $\mathrm{CuO}_{2}$ layers and is therefore a universal property of the superconducting $\mathrm{CuO}_{2}$ plane.

\section{Experimental}

Raman scattering experiments were performed on two different single crystals: the single layer $\mathrm{Hg}-1201$ $\left(\mathrm{T}_{c}=95 \mathrm{~K}\right)$ and the trilayer $\mathrm{Hg}-1223\left(\mathrm{~T}_{c}=125 \mathrm{~K}\right)$. Both crystals are very close to optimal doping and have tetragonal symmetry. The single crystal of $\mathrm{Hg}-1201$ has been successfully grown by the flux method. The detailed procedure for crystal growth will be described elsewhere [8]. Details on the synthesis of $\mathrm{Hg}-1223$ single crystals can be found in ref. [9]. Plane polarised spectra were taken using different lines from a $\mathrm{Ar}^{+}-\mathrm{Kr}^{+}$ laser and by a JY T64000 spectrometer (except for the Hg-1223 data where a JY U1000 was used). The spectra were corrected from the spectrometer response, the Bose factor and the optical constants thus yielding the imaginary part of the Raman response. Temperature indicated are corrected from the laser heating for $\mathrm{Hg}$ 1201 spectra (typically 10 to $15 \mathrm{~K}$ ) and are the nominal temperatures for $\mathrm{Hg}-1223$ spectra.

\section{Results and discussion}

\section{1. $\mathrm{Hg}-1201$}

In Figure 1 we show spectra in the $B_{1 g}, A_{1 g}$ and $B_{2 g}$ channels in the normal and superconducting states using the $2.4 \mathrm{eV}(514.5 \mathrm{~nm})$ excitation line. In the $B_{1 g}$ channel, which probes the antinodal region $((\pi, 0)$ points $)$, the response in the superconducting state shows the usual $2 \Delta_{0}$ pair breaking peak located around $520 \mathrm{~cm}^{-1}$. This gives a coupling ratio $\frac{2 \Delta_{0}}{k_{B} T_{c}}$ of 7.9. The response in the $A_{1 g}$ symmetry on the other hand shows a very strong superconductivity induced peak located below the $2 \Delta_{0}$ at an energy of $330 \mathrm{~cm}^{-1}$ $\left(\frac{E_{A_{1 g}}}{k_{B} T_{c}}=5.1\right)$. The presence of the two different energy scales is consistent with data on other optimally doped cuprates [5]. We note that the presence of a strong $A_{1 g}$ peak in a single layer cuprate rules out a scenario in which the peak is due to unscreened charge fluctuation between adjacent $\mathrm{CuO}_{2}$ layers. In the $B_{2 g}$ symmetry, which probes the nodal region, we do not observe any superconducting induced peak. The presence of a weak peak in the superconducting state of the $B_{2 g}$ symmetry appears to be material dependant and could be sensitive to disorder [10].

As was pointed out by Devereaux et al. [1] careful inspection of the power law behaviors in the $B_{1 g}$ and $B_{2 g}$

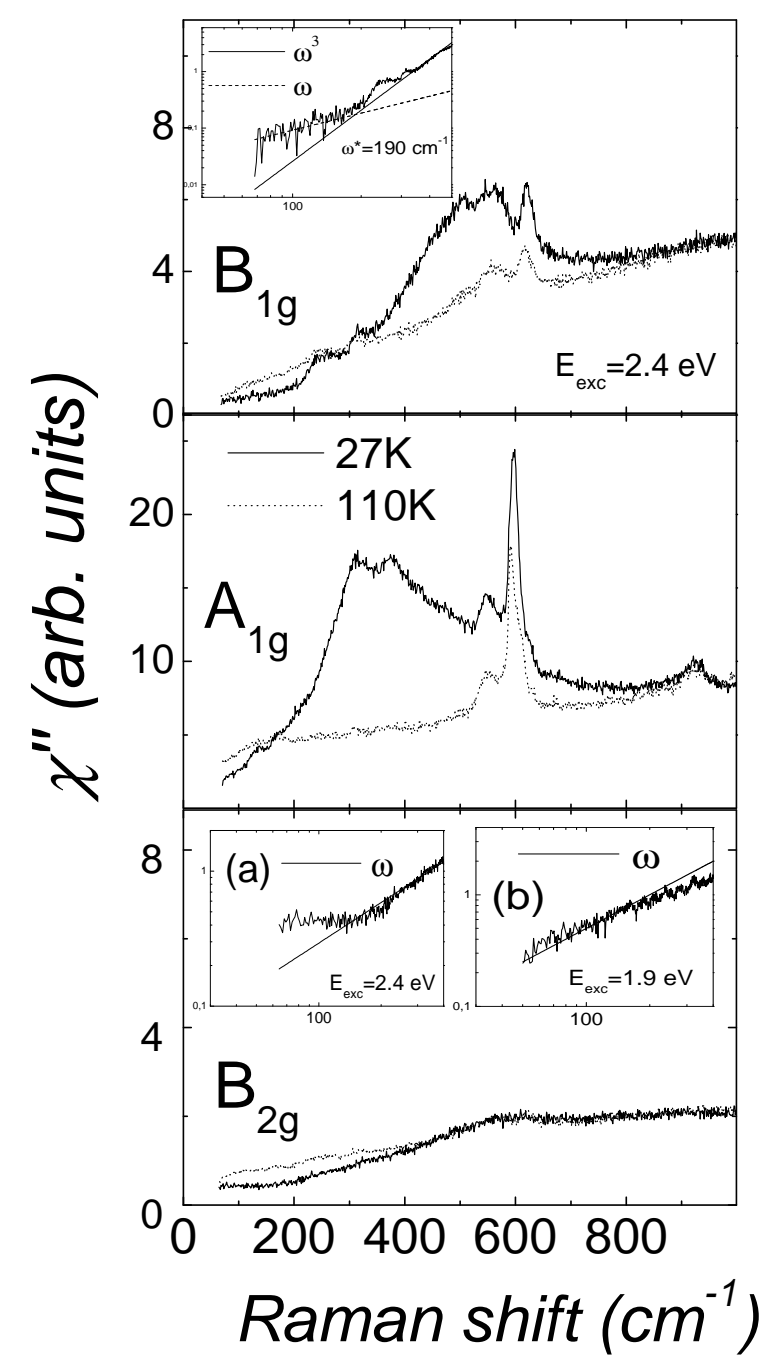

Fig. 1. Raman responses of optimally doped $\mathrm{Hg}-1201$ in $B_{1 g}$, $A_{1 g}$ and $B_{2 g}$ symmetries in the normal and superconducting states. The $A_{1 g}$ symmetry was obtained by substracting the $B_{2 g}$ component to the spectra taken in (x'x') scattering geometry $\left(A_{1 g}+B_{2 g}\right)$. Insets show log-log plots of the response in $B_{1 g}$ and $B_{2 g}$ symmetries with linear and cubic fits. All spectra are taken using the $2.4 \mathrm{eV}$ excitation line except spectrum shown in the inset (b) for the $B_{2 g}$ channel which was performed using the $1.9 \mathrm{eV}$ excitation line.

channels can yield information on the gap anisotropy. For a clean d-wave superconductor the low energy Raman response in $B_{1 g}$ symmetry is expected to follow a cubic law while the $B_{2 g}$ symmetry should be linear. In the $B_{1 g}$ channel a crossover between linear and cubic behavior is observed around $190 \mathrm{~cm}^{-1}$ (see inset) consistent with theoretical prediction for disordered d-wave superconductors [10]. Similar behavior in this symmetry using the $2.2 \mathrm{eV}$ and the $1.9 \mathrm{eV}$ excitation is observed (see Figures 2 and 3 ). On the other hand spectrum in the $B_{2 g}$ is linear except below $140 \mathrm{~cm}^{-1}$ where 


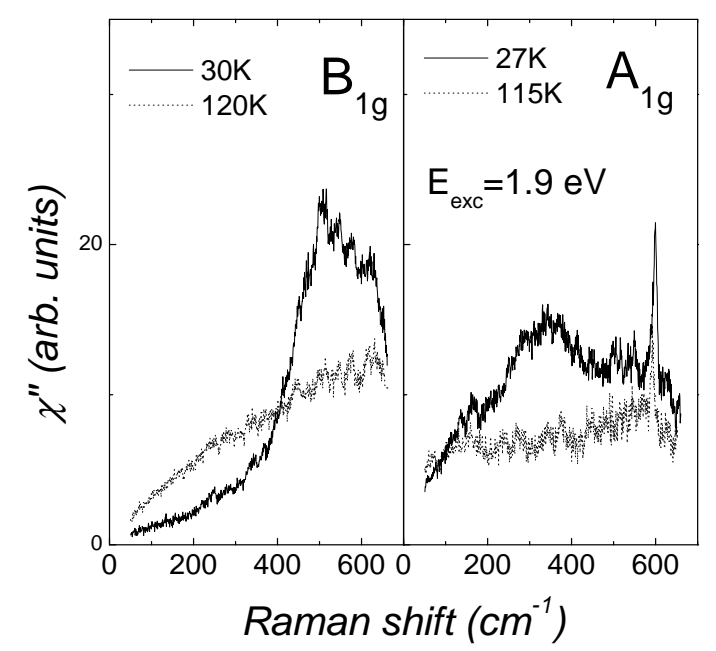

Fig. 2. Raman responses of $\mathrm{Hg}-1201$ in the $B_{1 g}$ and $A_{1 g}$ symmetries using the $1.9 \mathrm{eV}$ excitation line.

additional scattering is found producing a nearly flat continuum at low energy. The origin of this anomalous additional intensity is unclear but the same spectrum taken using the $1.9 \mathrm{eV}$ excitation energy display a linear behavior at low energy down to the lowest energy measured $\left(50 \mathrm{~cm}^{-1}\right)$ suggesting the presence of nodes in the $(\pi, \pi)$ direction in the gap function (see inset (b) of Figure 1). Thus the increase of scattering at low energy using the $2.4 \mathrm{eV}$ excitation is most likely spurious and we conclude that the present data indicate a gap of d-wave symmetry in optimally doped $\mathrm{Hg}-1201$.

In figures 2 and 3 we examine the resonant behavior of the $B_{1 g}$ and $A_{1 g}$ peaks. In Figure 2 we have plotted spectra in the normal and superconducting states performed using the $1.9 \mathrm{eV}$ excitation. We note that if both peak are located at the same energy as the one taken using the $2.4 \mathrm{eV}$, their relative intensities have changed drastically. While for the $2.4 \mathrm{eV}$ excitation the $A_{1 g}$ peak intensity more than twice the $B_{1 g}$ pair breaking peak intensity, the situation is opposite in the case of the $1.9 \mathrm{eV}$ excitation energy where the $A_{1 g}$ peak intensity is now a factor 1.5 smaller than the $B_{1 g}$ pair breaking peak. In Figure 3 the $B_{1 g}$ and $A_{1 g}$ channels in the superconducting state are shown as a function of the excitation energy used. Similarly to the case of Y-123 and Bi-2212 the $A_{1 g}$ peak position in $\mathrm{Hg}-1201$ is found to be insensitive to the choice of excitation energy and remains centered around $330 \mathrm{~cm}^{-1}$. Its intensity is nearly constant between $2.4 \mathrm{eV}$ and $1.9 \mathrm{eV}$ but is decreased by about a factor 2 when using the $2.2 \mathrm{eV}$ excitation line (see Figure 3 ). The $B_{1 g}$ pair breaking peak on the other hand shows a remarquable resonant behavior toward $1.9 \mathrm{eV}$ (see figure 3): its intensity is nearly the same for $2.4 \mathrm{eV}$ and $2.2 \mathrm{eV}$ but is enhanced for $1.9 \mathrm{eV}$ by more than a factor 4 . In a similar way the normal state $B_{1 g}$ continuum intensity is also enhanced

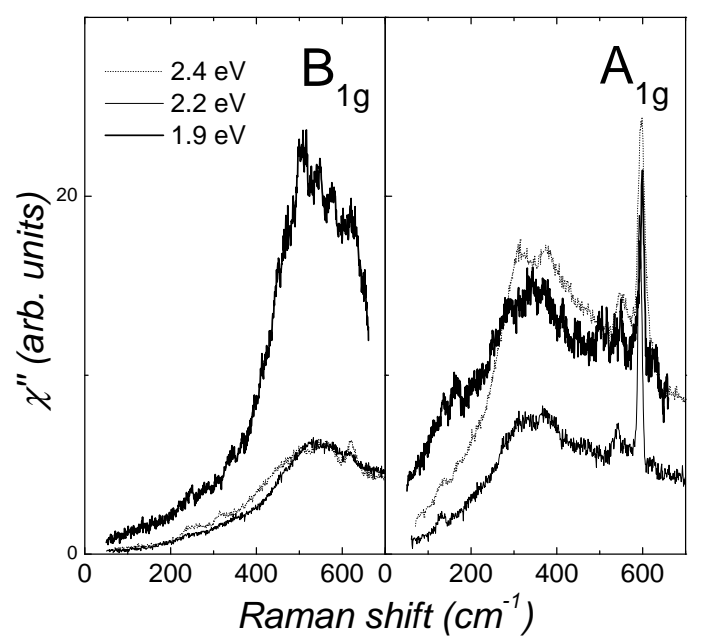

Fig. 3. $B_{1 g}$ and $A_{1 g}$ symmetries in the superconducting state $(\mathrm{T}=27 \mathrm{~K}-30 \mathrm{~K})$ taken at three different excitation energies.

towards $1.9 \mathrm{eV}$ (see Figures 1 and 2). Such a simultaneous resonant behavior in both the normal and superconducting states in the $B_{1 g}$ channel has also been observed in Tl2201 [11] and strongly advocates for an electronic origin of the normal state $B_{1 g}$ Raman continuum below $1000 \mathrm{~cm}^{-1}$.

Previous resonant Raman scattering studies have been mainly focused on the resonant properties of the $B_{1 g}$ (or $B_{2 g}$ ) pair breaking peak. Resonant Raman scattering data in Tl-2201 [11] show a strong increase of the $B_{1 g}$ peak intensity toward high excitation energies $(3 \mathrm{eV})$ and more recently resonant behavior of the $\mathrm{B}_{2 g}$ channel towards low excitation energy $(1.9 \mathrm{eV})$ was found in electron doped NCCO [12]. In a free electronlike picture of Raman scattering, resonant behavior is achieved when the incident (or scattered) photon energy equals an interband distance in k-space. In principle resonance effects can strongly affect the actual $\mathrm{k}$-dependance of the Raman vertices and thus be used to selectively probe different directions in $\mathrm{k}$-space [6]. In our case the resonance enhancement at $1.9 \mathrm{eV}$ in the $B_{1 g}$ channel indicates the presence of an interband transition near the $(\pi, 0)$ point of the Brillouin zone in $\mathrm{Hg}$-1201. If we believe band structure caculations, this transition most likely takes place between the non bonding bands and the Fermi level (antibonding band) [13].

We now discuss on the implications of the different resonant behaviors of the $B_{1 g}$ pair breaking peak and the $A_{1 g}$ peak. As mentionned in the introduction, theoretical Raman responses in a standard BCS d-wave framework systematically fail to reproduce the experimentally observed $A_{1 g}$ response. Probing essentially the whole Fermi surface the $A_{1 g}$ channel is expected to be greatly influenced by resonances. As a consequence, the $A_{1 g}$ peak position, if related to the amplitude of the 
superconducting gap, should be very sensitive to the choice of excitation energy, especially near resonances. In the present case, the resonant behavior of the Raman vertex in the $(\pi, 0)$ direction should result in a shift of the $A_{1 g}$ peak position towards $2 \Delta_{0}$ [6]. In contrast our data show that its position is constant despite the resonance effect in the $(\pi, 0)$ direction observed in the $B_{1 g}$ channel with the $1.9 \mathrm{eV}$ excitation line. Thus the $A_{1 g}$ peak is a true energy scale which is not directly related the amplitude of the d-wave superconducting gap. In fact near the optimally doped regime of many cuprates this energy scale tracks closely that of the neutron resonance observed in the spin excitation spectrum by INS $[5]$.

\section{2. $H g-1223$}

In figure 4 the $B_{1 g}$ and $A_{1 g}$ spectra in the superconducting state of $\mathrm{Hg}-1223$ are shown for the 1.9 and $2.4 \mathrm{eV}$ excitation lines. As in the case of $\mathrm{Hg}-1201$ the $B_{1 g}$ response shows a resonance toward $1.9 \mathrm{eV}$ but this time its intensity increases only by less than a factor 2 . The pair breaking peak position shifts slightly towards lower energy: from $2 \Delta_{0}=780 \mathrm{~cm}^{-1}=9.0 \mathrm{k}_{B} T_{c}$ for $2.4 \mathrm{eV}$ it moves down to $2 \Delta_{0}=720 \mathrm{~cm}^{-1}=8.3 \mathrm{k}_{B} T_{c}$ for $1.9 \mathrm{eV}$. These coupling ratios are very similar to the one measured in $\mathrm{Hg}-1201\left(2 \Delta_{0}=8.1 \mathrm{k}_{B} T_{c}\right)$ and suggest that the amplitude of the gap is controlled by $T_{c \max }$ in the $\mathrm{Hg}$ based cuprates family. A similar behavior was found in a ARPES study of the Bi family [14]. In the case of the $A_{1 g}$ channel the peak is located at $500 \mathrm{~cm}^{-1}=5.7 \mathrm{k}_{B} T_{c}$ (5.1 $k_{B} T_{c}$ for $\left.\mathrm{Hg}-1201\right)$. We also note that its intensity is the same for both excitation energies (again similar to the case of $\mathrm{Hg}-1201$, see Figure 3 ). The very similiar behavior between $\mathrm{n}=1$ and $\mathrm{n}=3$ in the $A_{1 g}$ channel with respect to both their resonance behavior and their scaling with $T_{c}$ rules out the possibility that the peak is dominated by a c-axis plasmon component in three and four layers cuprates as has been suggested recently [7]. On the contrary it clearly indicates that the $A_{1 g}$ peak has the same origin for both single and trilayer cuprates.

\section{Conclusion}

We have reported resonant Raman scattering in single layer Hg-1201. Analysis of the low energy part of the spectra in the superconducting indicates a gap of d-wave symmetry. Spectrum in the $B_{1 g}$ channel, contrary to the $A_{1 g}$ channel, shows a remarkable resonant behavior of the pair breaking towards the $1.9 \mathrm{eV}$ excitation energy. The different resonant behavior between both channels indicates different microscopic ori-

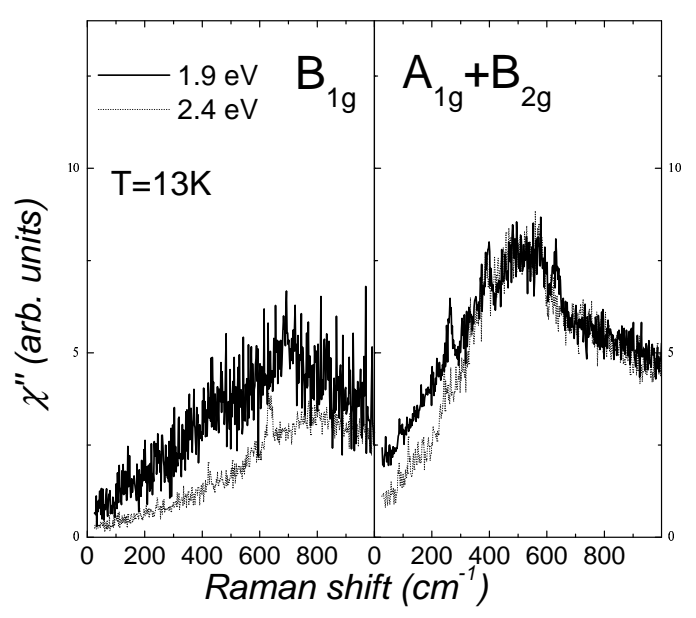

Fig. 4. Raman spectra of $\mathrm{Hg}-1223$ in the superconducting state in $B_{1 g}$ and $A_{1 g}+B_{2 g}$ symmetries at 1.9 and $2.4 \mathrm{eV}$. The $B_{2 g}$ componant is much weaker than the $A_{1 g}$ so that the $A_{1 g}+B_{2 g}$ spectrum is essentially dominated by the $A_{1 g}$ component.

gins for the $B_{1 g}$ pair breaking peak and the $A_{1 g}$ peak. The latter appears to be related to the neutron resonance energy scale. In addition, data on trilayer $\mathrm{Hg}$ 1223 demonstrate the universal character of the two energy scales, irrespective to the number of $\mathrm{CuO}_{2}$ layers. The presence in the $A_{1 g}$ Raman spectra of an energy scale also seen in the spin excitation spectrum by INS is presently not understood and recquires further experimental and theoretical investigations.

References

[1] T. P. Devereaux et al., Phys. Rev. Lett. 72, 396 (1994).

[2] A. Sacuto, J. Cayssol, D.Colson and P. Monod, Phys. Rev. B 61, $7122(2000)$.

[3] T. Strohm and M. Cardona, Phys. Rev. B 55, 12725 (1997).

[4] M. V. Klein and S. B. Dierker, Phys. Rev. B 29, 4976 (1984).

[5] Y. Gallais et al., Phys. Rev. Lett. 88177401 (2002).

[6] E. Sherman, C. Ambrosch-Draxl and O. V. Misochko, Phys. Rev. B 65, 140510 (2002).

[7] D. Munzar and M. Cardona, Phys. Rev. Lett. 90, 077001 (2003).

[8] D. Colson et al. to be published.

[9] D. Colson, A. Bertinotti, J. Hammann, J. F. Marucco and A. Pinatel, Physica C 233, 231 (1994).

[10] T. P. Devereaux, Phys. Rev. Lett. 74, 4313 (1995).

[11] M. Kang, G. Blumberg, M. V. Klein and N. N. Kolesnikov, Phys. Rev. Lett. 77, 4434, (1996).

[12] G. Blumberg et al., Phys. Rev. Lett. 88, 107002 (2002).

[13] C. Osvaldo Rodriguez, Phys. Rev. B 49, 1200 (1994). 
[14] T. Sato al., Phys. Rev. Lett. 89, 067005 (2002). 